\title{
Radar and Surface Measurement of Rainfall during CaPE: 26 July 1991 Case Study
}

\author{
EUGENIO GORGUCCI \\ Istituto di Fisica dell'Atmos(era (CNR), Rome, Ilaly \\ V. Chandrasekar \\ Colorado State University, Fort Collins, Colorado \\ GIANFRANCO SCARCHILLI \\ Istituto di Fisica dell'Atmos/era (CNR), Rome, Italy
}

(Manuscript received 18 January 1994, in final form 31 December 1994)

\begin{abstract}
Conventional usage of multiparameter radar measurements for rainfall estimation has been associated with tracking the variability of the raindrop size distribution. The use of multiparameter radar measurements in a statistical framework to estimate rainfall is presented in this paper. The techniques developed in this paper are applied to the radar and rain gauge measurement of rainfall observed on 26 July 1991, during the Convection and Precipitation Electrification program. Conventional pointwise estimates of rainfall are also compared. The probability matching procedure, when applied to the radar and surface measurements, shows that multiparameter radar algorithms can match the probability distribution functions better than the reflectivity based algorithms, thereby indicating the potential of multiparameter radar measurements for statistical approach to rainfall estimation.
\end{abstract}

\section{Introduction}

Remote estimation of rainfall is a topic of active research and was one of the topics of interest during the Convection and Precipitation Electrification program (CaPE) (Foote 1991). There have been two general approaches to estimate rainfall using radar, namely, (a) those that obtain an instantaneous point estimate in space and (b) those that provide climatological estimates over large areas. Most of the studies on the statistical techniques for obtaining mean estimate of rainfall use the reflectivity factor only (Calheiros and Zawadzki 1987), and a majority of the studies in the area of rainfall estimation using polarimetric techniques have concentrated on instantaneous spatial estimates of rainfall (Aydin et al. 1990). Conventionally, all the multiparameter researches have been associated with those studying the variability in raindrop size distribution (Seliga and Bringi 1976; Scarchilli et al. 1993). This paper presents for the first time the use of multiparameter radar data in a statistical framework. Extensive observation of multiparameter signatures in tropical and subtropical environments suggests that those storms are rich in polarimetric signatures (Bringi

Corresponding author address: Dr. Eugenio Gorgucci, Consiglio Nazionale della Ricerche, Istituto di Fisica dell'Atmosfera, P.le Luigi Sturzo, 31, 00144 Rome, Italy. et al. 1993). Therefore, inclusion of polarimetric signatures in a statistical rainfall estimation framework intuitively seems reasonable. This approach has the potential to avoid the typically nonlinear $\mathrm{dBZ}-\mathrm{dB} R$ ( $\log$ of rainfall rate) relationship that results from matching the radar and gauge CDFs (cumulative distribution function) of rainfall rate (Rosenfeld et al. 1993). Our paper uses a variation of the probability matching procedure in the context of multiparameter radar estimates of rainfall. A clear demonstration of improvements in rainfall estimates using multiparameter techniques over the conventional reflectivity-based estimates has been difficult due to the following reasons:

(a) There has never been a controlled experiment using multiparameter radar specifically designed to address the problem of rainfall estimation, and the scientists have had to work with datasets collected for other scientific objectives, and, therefore, with compromised scanning strategies.

(b) Until multiparameter procedures required good accuracy in radar measurements (to guarantee internal accuracy), precise calibration of radars was not monitored (up to 1-dB accuracy), resulting in the variation of $Z-R$ relations due to calibration errors.

Only a handful of previous experiments conducted very precisely were able to demonstrate the improvements in multiparameter rainfall estimates 


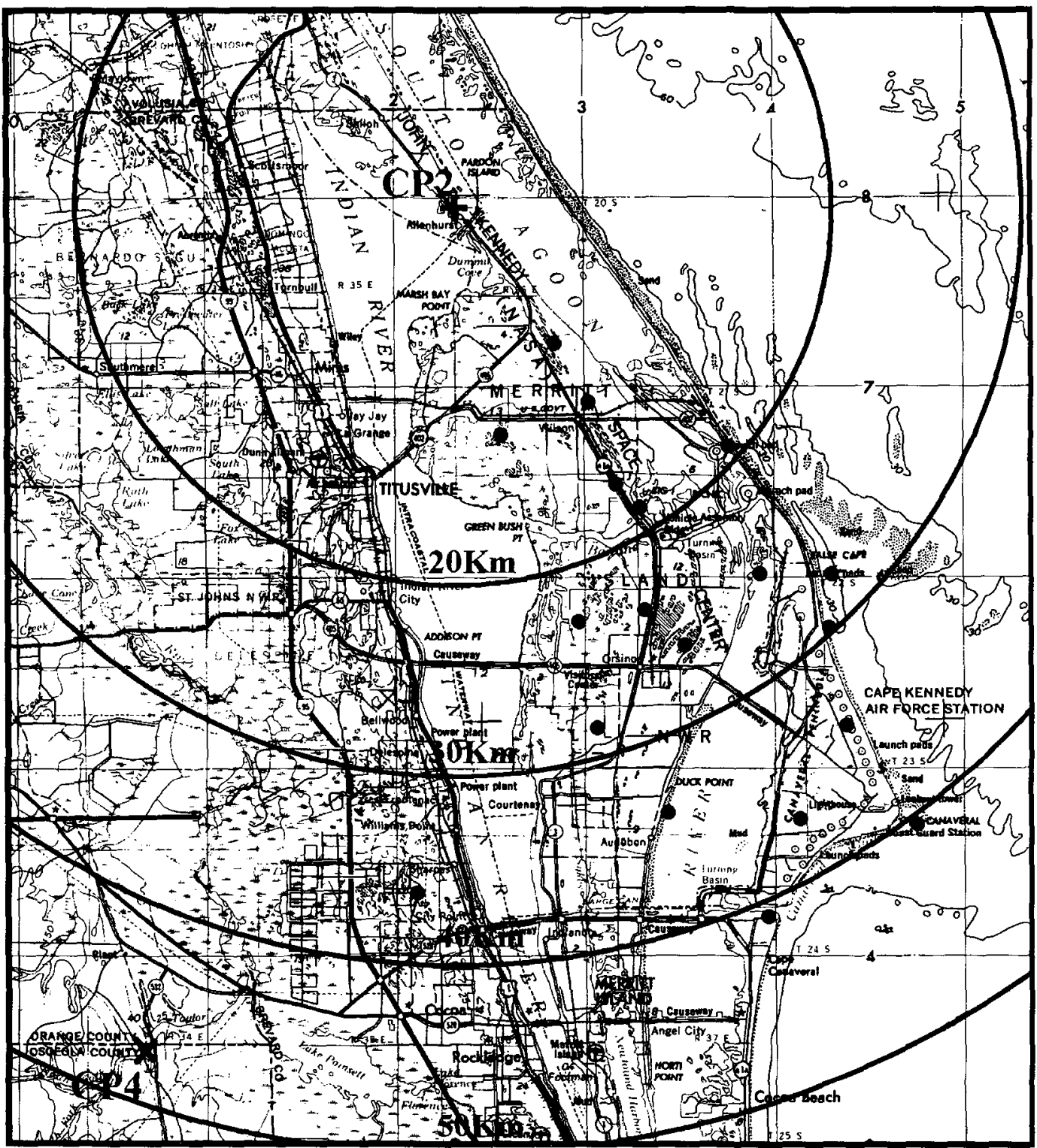

FIG. 1. The location of $\mathrm{CP}-2$ radar and rain gauge network during CaPE.

(Aydin et al. 1990). Even these experiments do not show improvements of the order predicted by theory owing to experimental limitations (Chandrasekar and Bringi 1988; Zawadzki 1984). Our approach does not treat the problem of multiparameter rainfall estimation as one that accounts for variability in drop size distribution (DSD) but treats it in a statistical framework, formulated in a way so that it can use the wealth of polarimetric signatures present in rainstorms. We have adapted the probability distribution function matching approach discussed by Calheiros and Zawadzki (1987) for multiparameter radar applications. We also present the conventional approach, attempting to compare point estimates of rainfall and study the contrast with statistical approach. In addition, we use the rainfall estimate obtained from the radar rainfall algorithms given by the probability matching procedure for comparison between radar and rain gauge at the TRMM (Tropical Rainfall Measuring Mission) rain gauge sites.

Our paper is organized as follows: section 2 describes the experimental setup during CaPE. In section 3 we compare the rainfall accumulation obtained at each rain gauge location with a similar estimate obtained from radar. Section 4 introduces the procedure for matching the probability density function of rainfall rates obtained by multiparameter radar and rain gauges. Section 5 summarizes the key results of this paper. 
TABLE 1. The characteristics of the CP-2 S-band radar.

\begin{tabular}{ll}
\hline \multicolumn{1}{c}{ Characteristic } & CP-2 S-band radar \\
\hline Polarization type & Linear \\
Wavelength $(\mathrm{cm})$ & 10.7 \\
Peak power $(\mathrm{kW})$ & 1200 \\
Pulse length $(\mu \mathrm{s})$ & 1.0 \\
PRF $\left(\mathrm{s}^{-1}\right)$ & 960 \\
Antenna type & Center fed paraboloid \\
Antenna size $(\mathrm{m})$ & 8.5 \\
Beamwidth & $0.93^{\circ}$ \\
Polarizations transmitted & Linear V or H \\
Polarization received & Copolar to transmit \\
Maximum sidelobe level $(\mathrm{dB})$ & -21 \\
Polarization control method & Ferrite switch \\
Polarization control period & Pulse by pulse \\
\hline
\end{tabular}

\section{CaPE rainfall measurement experiment}

CaPE was conducted in the central Florida region during the summer of 1991. One of the objectives of the CaPE program was remote estimation of rainfall by multiparameter radar. Florida's unique geographical and meteorological conditions contribute to the high frequency of thunderstorm activity during the summer. In the afternoon, the heated landmass is sufficiently large to produce deep convection. Furthermore, the action and the subsequent movement of the coastal sea breezes tend to focus this convection. During summer, the AtlanticBermuda high extends westward across central Florida. The north-south oscillations of the ridge tend to influence the locations and severity of thunderstorm development.

The sea-breeze circulation and the normal patterns of Florida convection assume different characteristics depending on whether the prevailing low-level flow has an onshore, offshore, or alongshore component. This larger-scale flow can accelerate or impede the daily progress of the sea breeze inland. Onshore flow (southwesterly flow) along the Gulf coast may drift eastward (usually in north-northeast-south-southwest lines) across the CaPE region. Generally, southwesterly flow is more unstable, contains deeper moisture, and more large-scale upward vertical motion than that from other quadrants. This regime can occur as much as $50 \%$ of the time during July and August, producing two-thirds of the storms in the CaPE region.

The instrumentation for rainfall measurement experiment during CaPE primarily consisted of the CP-2 multiparameter radar and a network of rain gauges called TRMM rain gauges located primarily in the vicinity of the Merrit Island area at the Kennedy Space Center (KSC). Figure 1 shows the location of the CP-2 radar and the TRMM rain gauge network.

The TRMM rain gauge network consists of 22 tipping-bucket rain gauges with a recording resolution of 1 min located at the National Aeronautics and Space Administration (NASA)/KSC and CaPE Canaveral Airforce Station (CCAFS). The parameters of interest for precipitation measurement that were measured by the $\mathrm{CP}-2$ radar were the reflectivity factor at horizontal polarization $\left(Z_{H}\right)$ and the differential reflectivity $\left(Z_{\mathrm{DR}}\right)$, both measured at $S$-band frequency. Table 1 lists the main features of the CP-2 radar that are relevant to this paper. Data used in this paper for rainfall measurement were collected, integrating 64 samples pairs with 1-ms PRT (pulse repetition time) in a PPI (plan position indicator) mode with a typical scan rate of $8^{\circ} \mathrm{s}^{-1}$.

26 July 1991 case study. On 26 July 1991 the CP-2 radar began operating early, and there was little activity during the morning. During the afternoon a cluster of storms moved over the TRMM rain gauge network, and the thunderstorm activity lasted for about $1 \mathrm{~h}$. The CP-2 radar collected data over these storms in a PPI mode obtaining data over the rain gauge network. Most of the rainfall activity discussed in this paper refers to a single precipitation event. Figure 2 shows a typical low-elevation PPI of the precipitation cell under study. We can see that the storm had high values of reflectivities ( $55 \mathrm{dBZ}$ ) producing significant rainfall over the TRMM network. Figure 3 shows the time record of rainfall rate at a typical gauge.

\section{Pointwise radar and rain gauge comparison}

\section{a. Data analysis procedure}

The procedure to compute point rainfall using radar and rain gauge is conceptually straightforward, but nu-

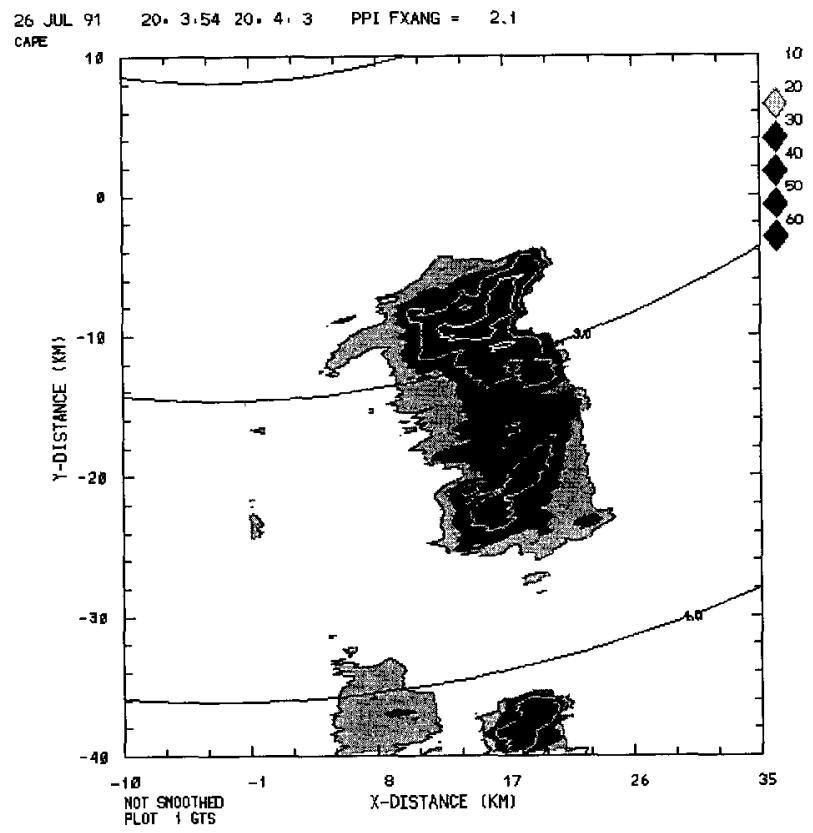

FiG. 2. A sample PPI of reflectivity observed on the afternoon of 26 July, during CaPE. The legend on the top of the contour plot is as follows: 20.3.54-20.4.3 indicates the time (UTC) when the volume of radar data was collected; PPI FXANG $=2.1$ indicates that the PPI scan was done at fixed elevation angle of $2.1^{\circ}$; the gray-scale patterns indicate the contour levels. 


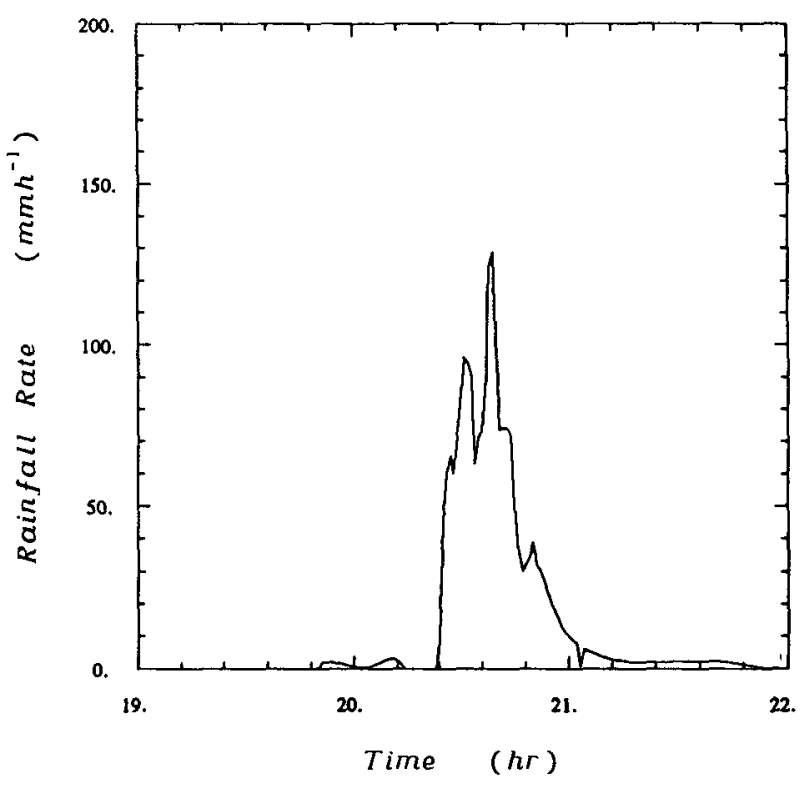

FIG. 3. Sample time record of a TRMM rain gauge during the afternoon of 26 July (time in UTC).

merous details are important. We want to stress here that we have not made any adjustment for storm movement, or any other adjustment or fine-tuning of the data, and the comparison is direct. The actual steps involved in collecting radar data for each rain gauge location is as follows. (a) The location of each rain gauge is mapped on the radar PPI of reflectivity factor and differential reflectivity. Subsequently, the radar data are converted to rainfall rate using each of the following three algorithms:

(i) the Marshall-Palmer (1948) relation

$$
R\left(Z_{H}\right)=0.0365 Z_{H}^{0.625},
$$

(ii) the GARP Tropical Atlantic Experiment (GATE) relation (Hudlow et al. 1979)

$$
R\left(Z_{H}\right)=0.0129 Z_{H}^{0.8}, \text { and }
$$

(iii) the dual-polarization algorithm (Gorgucci et al. 1993)

$$
R_{\mathrm{DR}}=10 \times 10^{-3} Z_{H}^{0.92} 10^{-0.369 Z_{\mathrm{DR}}} .
$$

(b) The radar estimates are then averaged over their nearest neighbors within $1 \mathrm{~km}$ on each side. This is done to smooth the data over measurement errors. We note here that in the above equations $Z_{H}$ has its standard units $\left(\mathrm{mm}^{6} \mathrm{~m}^{-3}\right)$ and $Z_{\mathrm{DR}}$ is in decibels. The rainfall obtained from radar over time is then accumulated, at each rain gauge location, for the entire precipitation event, and the results are discussed in the following section.

\section{b. Experimental results}

Rainfall accumulation at each rain gauge location is estimated for comparison with rain gauge estimates. The rainfall accumulation is estimated using the three algorithms given by (1), (2), and (3), namely, two $Z$ $R$ relations and the one given by $Z_{H}$ and $Z_{\mathrm{DR}}$. Figure 4 shows the comparison of rainfall accumulation at the TRMM rain gauge sites in the form of scatterplot. In Fig. 4 the square points denote Marshall-Palmer rainfall estimates, the open circles denote the GATE algorithm, and the diamond symbols indicate dual polarization rainfall algorithm. We have also estimated a figure of merit to quantitatively describe the capability of algorithms to estimate rainfall, namely, the fractional standard error (FSE) defined as

$$
\mathrm{FSE}=\frac{\left\{\frac{1}{N} \sum[\text { rainfall (radar) }\right.}{\left.- \text { rainfall (gauge) }]^{2}\right\}^{1 / 2}},
$$

where $N$ represents the number of rain gauges. The averages are computed using data from several rain gauge locations. The FSE of the MarshallPalmer rainfall estimate was found to be $49 \%$, whereas the standard error in the GATE $Z-R$ relation was found to be $58 \%$. The corresponding standard error in dual polarization rainfall estimate was $35 \%$.

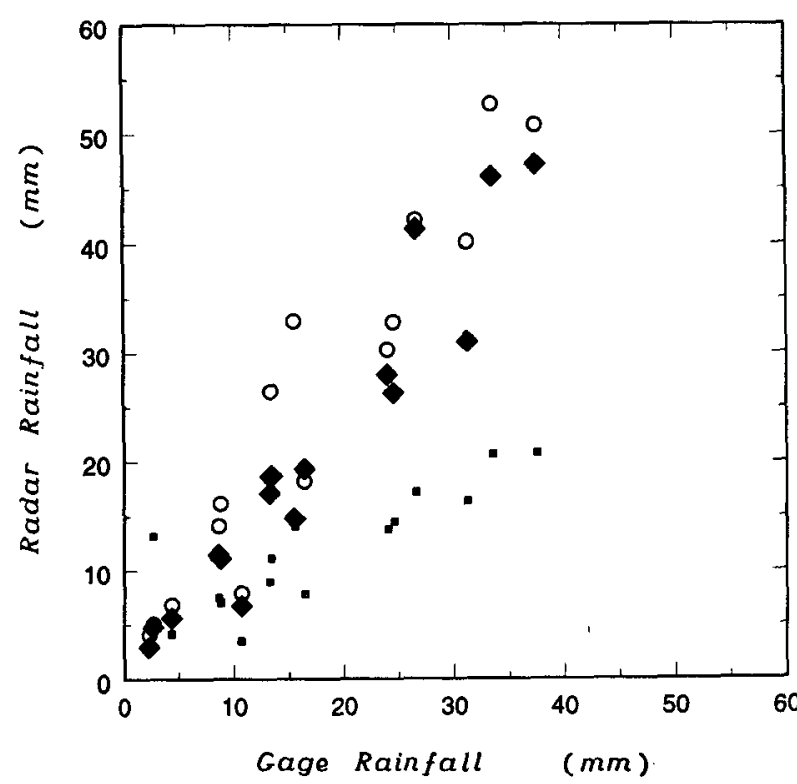

FIG. 4. The radar and rain gauge rainfall accumulation observed at the TRMM rain gauge sites. The radar-based rainfall is computed using three algorithms, namely, the Marshall-Palmer $Z-R$ relation $(\square)$, the GATE $Z-R$ relation $(O)$, and the dual polarization algorithm (ब). 


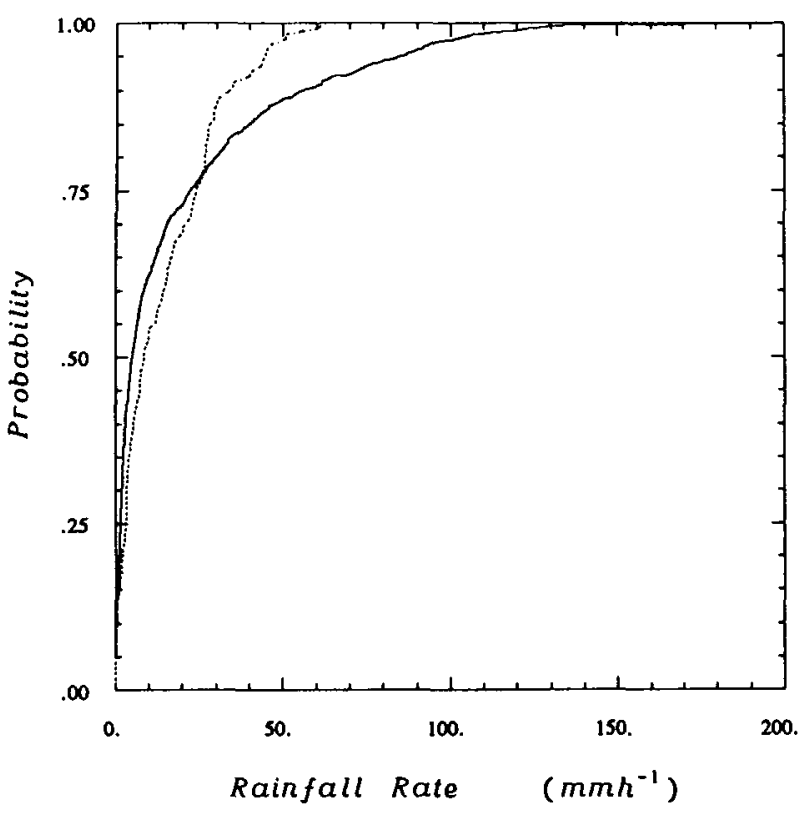

FIG. 5. The experimental probability distribution function (CDF) of rainfall. The solid line shows the CDF observed by rain gauge, whereas the dotted line shows the CDF obtained from radar data using the Marshall-Palmer $Z-R$ relation.

The intent of this section is not to study the pointwise performance of the multiparameter relation with the GATE or Marshall-Palmer $Z-R$ relation. However, with the presentation of these results, we set the stage for subsequent analysis in the rest of the paper. One point that is worth noting is that the multiparameter relation is theoretically obtained (based on a generalized gamma DSD model ). The multiparameter rainfall estimates perform better than the two $Z-R$ rainfall algorithms evaluated here; however, at this stage we are not sure whether these are the best $Z-R$ relation, or whether the multiparameter estimate can be better than the best $Z-R$ relation that can be derived. All of the above considerations are discussed in the following sections. One conclusion that can be reached from the results of Fig. 4 is that in the absence of the knowledge of the best $Z-R$ relation to use, the multiparameter radar estimates seem to outperform a conventional $Z$ $R$ relation. This is one of the few results that have been demonstrated based on data (see also Aydin et al. 1990).

In the following section we evaluate procedures to obtain parameters of the best $Z-R$ relation and multiparameter rainfall relation. We study the probability distribution function (CDF) matching procedure for evaluating the best parametric relation based on radar and rain gauge data.

\section{Parametric estimates based on cumulative distribution function of rainfall rate}

The CDF of rainfall can be constructed from radar and the functional shape will depend on the type of algorithm used to convert the radar observations to rainfall. The rainfall conversion algorithm can be $Z$ $R$ based or multiparameter based depending on the algorithm used. The $Z-R$ algorithms have parameters of the following form:

$$
R\left(Z_{H}\right)=C_{1} Z_{H}^{\alpha},
$$

where $C_{1}$ and $\alpha$ are parameters of the algorithm. Similarly, the multiparameter rainfall estimate $R\left(Z, Z_{\mathrm{DR}}\right)$ has the form

$$
R\left(Z, Z_{\mathrm{DR}}\right)=C_{2} Z^{\beta} 10^{-\mu Z_{\mathrm{DR}}},
$$

where $C_{2}, \beta$, and $\nu$ are the parameters of the algorithm. Our procedure for estimating the parameters of the radar rainfall algorithm based on CDF matching is as follows:

1) For a given starting guess of parameters $C_{1}, \alpha$, $C_{2}, \beta$, and $\nu$, evaluate the radar rainfall estimate at each rain gauge location as described in section 3. Typically, the initial guess is based on an established relation such as the Marshall-Palmer $Z-R$ relation or the GATE $Z-R$ relation.

2) Construct the CDF based on result of step 1.

3 ) Construct the CDF based on rain gauge observations.

4) Construct the mean-square error (ERR) between the radar-based and rain gauge-based CDF, obtained as the integration of the square deviation between the two CDFs over the entire range.

5) Iterate the coefficients to minimize the meansquare error.

Figures 5, 6, and 7 show comparisons of the CDF of rainfall obtained from rain gauge and radar using the Marshall-Palmer $Z-R$ relation, the GATE $Z-R$ relation, and the multiparameter algorithm given by (3), respectively. The corresponding mean-square error between the two CDFs in self consistent units is as follows:

(a) Marshall-Palmer $Z-R$ equation: $\mathrm{ERR}=236.7$

(b) GATE $Z-R$ equation: ERR $=200.3$

(c) multiparameter rainfall algorithm (3): ERR $=129.5$,

where ERR is the mean-square error defined in step 4.

The above analysis based on the CDF of rainfall rate again suggests that in absence of the knowledge of the right kind of $Z-R$ relations to use, multiparameter radar rainfall algorithm performs better than $Z-R$ relations. We proceed next to obtain the optimum $Z-R$ and multiparameter relations. We must note here that our optimization procedure is done using nonlinear optimization algorithms. We do not linearize the $Z$ $R$ relation or $R\left(Z_{H}, Z_{\mathrm{DR}}\right)$ taking logarithms. The linearization procedure taking logarithms disturbs the natural distribution of rainfall in the minimization 


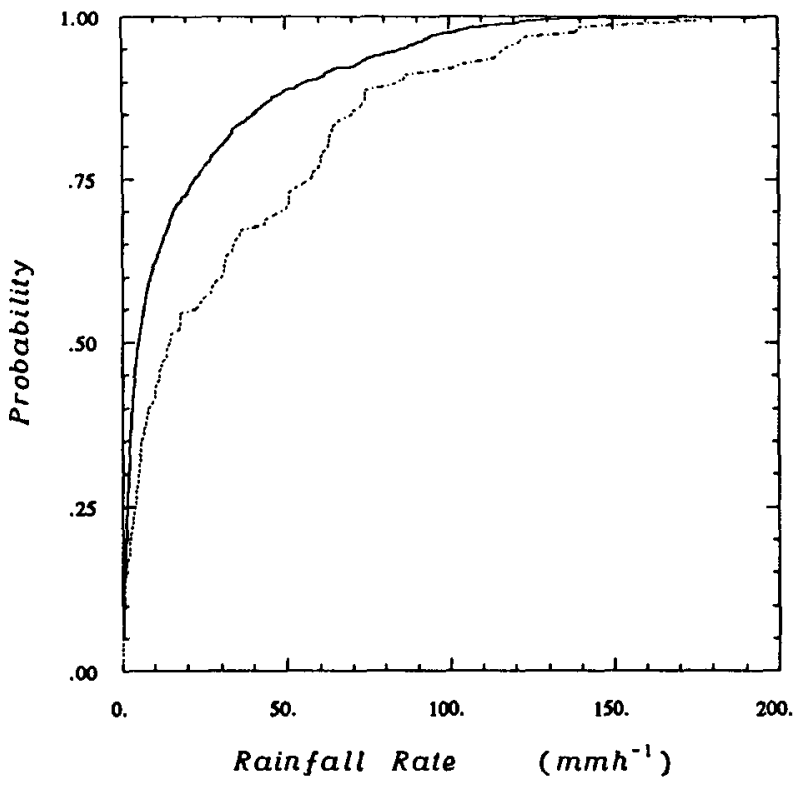

FIG. 6. Same as Fig. 5 except the radar-based CDF of rainfall is obtained using the GATE $Z-R$ relation.

process. The resulting parameter estimates and error variance for $R\left(Z_{H}\right)$ and $R\left(Z_{H}, Z_{\mathrm{DR}}\right)$ based on CDF matching criteria are as follows:

$$
R\left(Z_{H}\right)=123.5 \times 10^{-3} Z_{H}^{0.572}
$$

with the corresponding mean-square error ERR $=195.1$, and

$$
R\left(Z_{H}, Z_{\mathrm{DR}}\right)=10 \times 10^{-3} Z_{H}^{0.914} 10^{-0.377 Z_{\mathrm{DR}}}
$$

with the corresponding mean-square error ERR $=114.4$.

There are three important observations we can make from the results depicted in (7) and (8). First, the optimum $Z-R$ relation matching the CDF is different from both the GATE and Marshall-Palmer algorithms. Second, the best multiparameter algorithm based on $\left(Z_{H}, Z_{\mathrm{DR}}\right)$ has a lower mean-square error compared to the best $Z-R$ relation, thereby indicating that in a statistical approach to rainfall estimation, polarimetric techniques have some improvement to offer. Another possibly more significant conclusion of practical importance is the optimum $R\left(Z_{H}, Z_{\mathrm{DR}}\right)$ has parameters and error structure very close to the theoretical algorithm, indicating thereby that we can possibly do with just one expression. It is to be noted here that we have followed a new approach to obtain polarimetric radar estimates of rainfall. Historically, polarimetric radar techniques have all been branded as those that solve for variabilities in raindrop size distribution. However, in this paper we have tried to address a different question. Polarimetric radars provide alternate measurements, compared to reflectivity factor, that have different statistical spatial and temporal covariance struc- ture (Krajewski et al. 1993). Therefore, polarimetric measurements have the potential to add additional information in the context of rainfall estimation. We have evaluated whether the polarimetric measurements of rainstorms have something to add "in a statistical sense" to the problem of rainfall estimation. The preliminary answer seems to be "yes."

The algorithms given by (7) and (8) have been obtained in a statistical framework for radar-rain gauge comparison. In the following, we compare the estimation of rainfall accumulation at the TRMM gauge locations based on (7) and (8) for point comparisons, of the type done in section 3 .

Figures 8 and 9 show the scatterplot of rainfall accumulation at TRMM rain gauge locations based on $R\left(Z_{H}\right)$ and $R\left(Z_{H}, Z_{\mathrm{DR}}\right)$ obtained using the procedure described in section 3 but substituting the recomputed algorithms (7) and (8). The standard error of Fig. 8 based on $R\left(Z_{H}\right)$ is $25 \%$, whereas the corresponding standard error for $R\left(Z_{H}, Z_{\mathrm{DR}}\right)$ is $21 \%$. Both the error percentages are artificially low because the minimization and error computation are done on the same dataset that was used to obtain the parameters. However, even under such conditions, the best $R\left(Z_{H}, Z_{\mathrm{DR}}\right)$ seems to have a slight edge over the best $R\left(Z_{H}\right)$. Though the optimization criteria for parameter estimation is different from the point estimate comparison, the results seem similar. We can also obtain optimum estimates of coefficients to use in $R\left(Z_{H}, Z_{\mathrm{DR}}\right)$ based on direct pointwise matching of rainfall accumulation itself as the criterion. In other words, we minimize the FSE defined in (4) to obtain the optimum parameters in (6). We perform the minimization directly on the

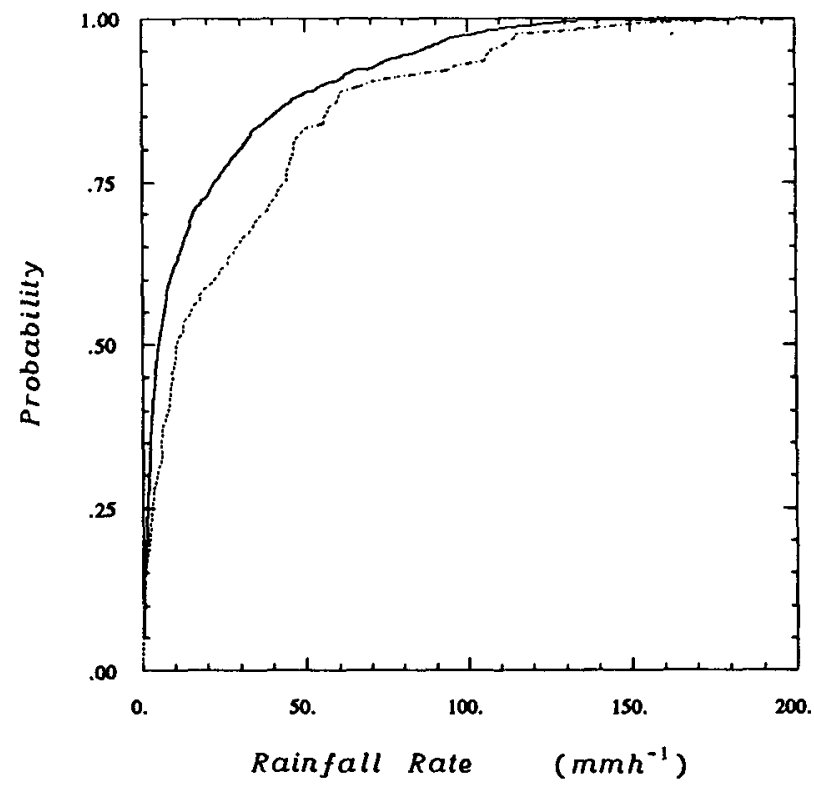

FIG. 7. Same as Fig. 5 except the radar-based CDF of rainfall is obtained using the dual-polarization algorithm. 


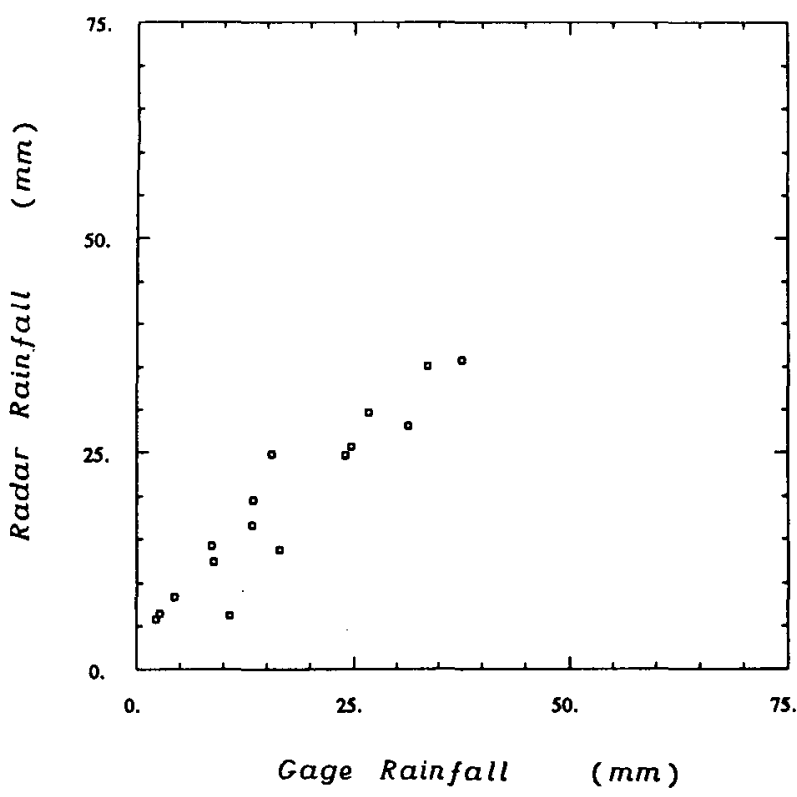

FIG. 8. Scatterplot of rainfall accumulation observed by rain gauge and radar at the TRMM rain gauge sites. The radar-based rainfall accumulation is computed using the $Z-R$ relation that provides the best matching between the observed CDF of rainfall by radar and rain gauges.

mean-square error between the cumulative rainfall estimates from radar and rain gauge. The parameterization that gives the least fractional standard error defined by $(4)$ is

$$
R_{\mathrm{DR}}=10 \times 10^{-3} Z_{H}^{0.91} 10^{-0.385 Z_{\mathrm{DR}}} .
$$

We can see that the above parameterization compares well with (8) and (3). In summary, the $R_{\mathrm{DR}}$ parameterizations obtained by two independent criteria agree with each other and the theoretical relation available in the literature.

\section{Summary and conclusions}

Two general approaches to remotely estimate rainfall with radar are studied here:

(a) obtain instantaneous point estimates of rainfall;

(b) apply statistical techniques in a mean sense for the whole storm, or climatological region.

Conventionally, multiparameter radar estimates of rainfall take the first approach and are primarily associated with accounting for the variability in the drop size distribution, whereas the statistical techniques use only reflectivity. This paper presents for the first time the use of multiparameter radar measurements in a statistical framework for estimating rainfall. We also compare pointwise rainfall estimation using $Z-R$ as well as multiparameter algorithms. We used data from a rain event during $\mathrm{CaPE}$ to test the analytical procedures developed in this paper. Pointwise comparison of rainfall accumulation at the TRMM rain gauge sites using multiparameter estimate $R_{\mathrm{DR}}$ was found to have a standard error of $35 \%$, whereas the GATE and MarshallPalmer $Z-R$ relations were found to have a standard error of $58 \%$ and $49 \%$, respectively.

We have analyzed the difference between the experimental CDF obtained from radar and rain gauge network. Such an analysis showed that Marshall-Palmer and GATE $Z-R$ relations had a mean-square error (in its own internally self-consistent units) of 256 and 200 , respectively, whereas the theoretical $R_{\mathrm{DR}}$ had an error of 129.5 . We subsequently obtain parameterization for the $R_{\mathrm{DR}}$ and $Z-R$ algorithms matching the experimental CDF of rainfall from radar to the one that is observed by rain gauge. This analysis showed that $R_{\mathrm{DR}}$ can be parameterized to obtain better a match between the experimental CDF of rainfall obtained by radar and rain gauge network (with an error of 114). The best match between the radar and rain gauge CDF was obtained for an $R_{\mathrm{DR}}$ relation not very different from the theoretical relation given by (3). In addition, the best $Z-R$ relation defined as the one that will minimize the difference between radar and rain gauge network-based CDFs had a meansquare error of 195 (in self-consistent units) higher than the theoretical $R_{\mathrm{DR}}$ relation. In summary, it appears from this preliminary analysis that the theoretical $R_{\mathrm{DR}}$ algorithm can match the CDF observed by rain gauge network, even better than the best $Z-R$ relation that can be determined after the fact (i.e., the $Z-R$ relation that fits the data best).

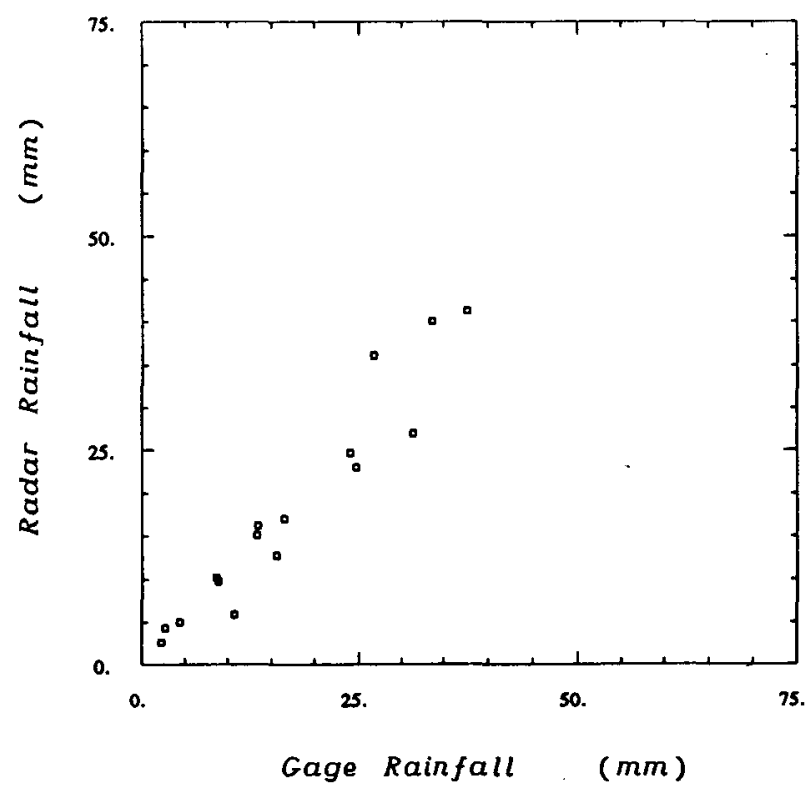

FIG. 9. Same as Fig. 8 except the radar-based rainfall accumulation is computed using the dual-polarization algorithm that provides the best matching between the observed CDF of rainfall by radar and rain gauges. 
Our preliminary results indicate that multiparameter radar measurements of rainfall can be successfully used in a statistical framework. It also appears from our analysis that multiparameter radar measurements show good potential for use in the context of statistical procedures for rainfall estimation. These are only demonstrations of initial observations, and we recognize that extensive study is necessary to evaluate our preliminary conclusions.

We want to add one concluding note about the importance of absolute calibration. The importance of absolute calibration cannot be overstated. A couple-of-decibels error in absolute calibration can easily go unnoticed during the experiment, showing no obvious symptoms; however, this can have serious consequences on the measurement of rainfall. All the procedures that even remotely involve the reflectivity factor will fail in the presence of an error in absolute gain of the radar system. This will have unintended consequences such as creation of a new $Z-R$ relation.

Acknowledgments. This research was supported by the EEC Program on the Environment "Storm '93" (PL910060: EC-1991-94, IFA-CNR), NASA, and the NSF(ATM-9200761). The CaPE program was a cooperative multiagency field program sponsored by the National Science Foundation (NSF), Federal Aviation Administration (FAA), National Aeronautics and Space Administration (NASA), National Oceanic and Atmospheric Administration (NOAA), and the United States Airforce (USAF). The authors acknowledge the assistance from Dr. Bill Crosson in obtaining the rain gauge data and Mr. Pasquale Iacovelli in the preparation of the paper.

\section{REFERENCES}

Aydin, K., Y. M. Lure, and T. A. Seliga, 1990: Polarimetric radar measurements of rainfall compared with ground-based rain gauges during MAYPOLE '84. IEEE Trans. Geosci. Remote Sens., 28, 443-449.

Bringi, V. N., A. Detwiler, V. Chandrasekar, P. L. Smith, L. Liu, I. J. Caylor, and D. Musil, 1993: Multiparameter radar and aircraft study of the transition from early to mature storm during CaPE: The case of 9 August 1991. Preprints, 26th Conf. on Radar Meteorology, Norman, OK, Amer. Meteor. Soc., 318-320.

Calheiros, R. V., and I. Zawadzki, 1987: Reflectivity-rain rate relationships for radar hydrology in Brazil. J. Climate Appl. Meteor., 26, 118-132.

Chandrasekar, V., and V. N. Bringi, 1988: Error structure of multiparameter radar and surface measurements of rainfall. Part I: Differential reflectivity. J. Atmos. Oceanic Technol., 5, 783795.

Foote, G. B., 1991: Scientific overview and operations plan. National Center for Atmospheric Research, Boulder, CO, 145 pp.

Gorgucci, E., G. Scarchilli, and V. Chandrasekar, 1993: A robust pointwise estimator of rainfall rate and liquid water content using polarization diversity radar. Preprints, 26th Conf. on Radar Meteorology, Norman, OK, Amer. Meteor. Soc., 777-779.

Hudlow, M. R., R. Arkell, V. Patterson, P. P. Pylowany, F. Richards, and S. Geotis, 1979: Calibration and intercomparison of the GATE C-band radar. NOAA Tech. Rep. EDIS 31, U.S. Dept. of Commerce, Washington, DC, $98 \mathrm{pp}$.

Krajewski, W. F., R. Raghavan, and V. Chandrasekar, 1993: Physically based simulation of radar rainfall data using a space-time rainfall model. J. Appl. Meteor., 32, 268-283.

Marshall, J. S., and W. M. Palmer, 1948: The distribution of raindrops with size. J. Meteor., 10, 25-29.

Rosenfeld, D., D. B. Wolff, and D. Atlas, 1993: General probabilitymatched relations between radar reflectivity and rain rate. $J$. Appl. Meteor., 32, 50-72.

Scarchilli, G., E. Gorgucci, V. Chandrasekar, and T. A. Seliga, 1993: Rainfall estimation using polarimetric techniques at C-band frequencies. J. Appl. Meteor., 32, 1150-1160.

Seliga, T. A., and V. N. Bringi, 1976: Potential use of the radar reflectivity at orthogonal polarizations for measuring precipitation. J. Appl. Meteor., 15, 69-76.

Zawadzki, I., 1984: Factors affecting the precision of radar measurements of rain. Preprints, 22d Conf. on Radar Meteorology, $\mathrm{Zu}-$ rich, Switzerland, Amer. Meteor. Soc., 251-256. 